\title{
Rapid High Mass Resolution Mass Spectrometry using Matrix-Assisted Ionization
}

\author{
Sarah Trimpin, ${ }^{1,2 *}$ Daniel W. Woodall, ${ }^{1}$ Shameemah Thawoos, ${ }^{1}$ Casey D. Foley, ${ }^{1}$ \\ Jing Li, ${ }^{1}$ Ellen D. Inutan, ${ }^{1}$ and Paul M. Stemmer ${ }^{3}$ \\ ${ }^{1}$ Department of Chemistry, Wayne State University, Detroit, Michigan 48202, USA \\ ${ }^{2}$ Cardiovascular Research Institute, Wayne State University School of Medicine, Detroit, MI, \\ 48202, USA \\ ${ }^{3}$ Proteomics Core Facility, Institute of Environmental Health Sciences, Wayne State University, \\ Detroit, Michigan 48202, USA
}

*Corresponding author: strimpin@chem.wayne.edu

\begin{abstract}
Matrix-assisted ionization (MAI) is demonstrated to be a robust and sensitive analytical method capable of analyzing proteins such as cholera toxin B-subunit and Pertussis toxin mutant from conditions containing relatively high amounts of inorganic salts, buffers, and preservatives without the need for sample clean up or concentration beforehand. By circumventing some of the sample preparation steps, MAI simplifies and speeds up the analytical workflow for biological samples in complex media. The benefits of multiply charged ions characteristic of electrospray ionization (ESI) and the robustness of matrix-assisted laser desorption/ionization (MALDI) can be obtained from a single method, making it well suited for analysis of proteins and other biomolecules at ultra-high resolution as is shown on an Orbitrap Fusion where protein subunits were resolved for which MALDI-TOF failed. MAI results are compared with those obtained with ESI, MALDI, and laserspray ionization methods and fundamental commonalities discussed.
\end{abstract}


Graphical Abstract:

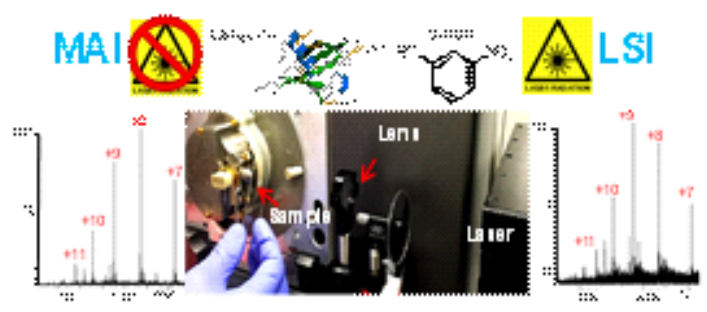

Highlights:

- Matrix-assisted ionization (MAl) from surfaces with and without laser ablation.

- Membrane-associated proteins ionized from buffer solutions for mass spectrometry (MS) analysis.

- Rapid protein analysis from high salt content samples using MAl at (ultra) high resolution

- Comparison of MAI, ESI, and MALDI for analyzing cholera toxin directly from buffer.

- MAI analysis of hexameric pertussis toxin from buffer showing protein subunits.

Keywords: matrix-assisted, laserspray, ionization, protein, mass spectrometry, high resolution

\section{Introduction}

Mass spectrometry (MS) has been established as a prominent analytical method for the study of biomolecules, particularly in the analysis of proteins [1]. Analysis of membraneassociated proteins is of significant interest because of their role in the function of many drugs and biomolecules [2]. Matrix-assisted laser desorption/ionization (MALDI) and electrospray ionization (ESI) are soft ionization methods widely used in the analysis of biomolecules because of their ability to accurately identify them from a variety of media. ESI generates multiply charged analyte ions from solution by applying a high voltage, whereas MALDI generates predominantly singly charged molecules from solid-phase matrix:analyte crystals with the use of a laser. Matrix-assisted ionization (MAI) is a recently introduced [3] ionization method that generates ESI-like multiply charged ions, but from solid-phase matrix:analyte crystals similar to MALDI. Contrary to ESI and MALDI, MAI does not require the use of high voltage or a laser to 
generate ions, using only the inherent vacuum [4] of the mass spectrometer and a MAI matrix [5]. The production of multiply charged analyte ions shifts the observed signals to a lower massto-charge ratio $(\mathrm{m} / \mathrm{z})$, allowing MAI to be used with atmospheric pressure (AP) high-performance mass spectrometers that have a limited mass range but improved mass resolution [6-9]. MAI is operational from both $\mathrm{AP}$ and intermediate pressure with only minor or no instrument modifications aside from removing the AP source housing and overriding interlocks [10]. Laserspray ionization (LSI), is a special case of MAI, in which a laser is used to ablate matrix:analyte crystals into the AP inlet of the mass spectrometer where ionization is initiated [11-14].

Biological samples commonly contain high concentrations of inorganic salts, as well as buffers to enhance solubility and stabilize $\mathrm{pH}$. These constituents suppress ionization in MS partially due to charge competition, salt adduction, and reduced analyte desolvation/declustering [15], most noticeably with ESI. For this reason sample purification, desalting, and concentration methods such as reverse-phase liquid chromatography (LC) [16], solid phase extraction [17], or chip-based microfluidic devices [18] are often used to reduce the sample complexity prior to ionization. Commonly used buffers in biochemistry such as TRIS and phosphate salt buffers are not compatible for use with ESI, and high salt content can cause clogging of the electrospray capillary [19]. As a result, a buffer exchange step is often required prior to analysis. While these methods are effective, they are time consuming and usually result in loss of analyte during sample preparation.

Predominantly multiply charged protonated analyte ions are observed using MAI, even in the presence of inorganic salts and sample preservatives such as ethlyenediaminetetraacetic acid (EDTA). MAI is demonstrated here for proteins, including membrane-associated bacterial toxins, for which a direct and robust method, capable of ionizing proteins from salty or buffered solutions without sample preparation or cleanup is desirable. 


\section{Experimental}

\section{Mass Spectrometers}

Analyses were performed on Waters SYNAPT G2 (Manchester, UK) and SYNAPT G2S mass spectrometers with the ESI housing removed and interlocks overridden as previously described [10]. The temperature of the Waters Z-Spray source block was typically set between 30 and $50^{\circ} \mathrm{C}$ to achieve maximum sensitivity [20]. Thermo LTQ Velos and Orbitrap Fusion (San Jose, CA) mass spectrometers were also used but typically operated at $150{ }^{\circ} \mathrm{C}$ and $100{ }^{\circ} \mathrm{C}$, respectively. ESI was performed at a flow rate of $10 \mu \mathrm{L} \mathrm{min}-1$ and at a capillary voltage at 3.0 kV. MALDI analyses were performed on a Bruker MALDI-TOF Ultraflextreme II (Bremen, Germany).

\section{Materials and Sample Preparation}

Cholera toxin B-subunit (11.6 kDa), myoglobin (16.7 kDa), cytochrome C (12.0 kDa), calmodulin (16.7 kDa), lysozyme (14.3 kDa), ubiquitin (8.5 kDa), bovine insulin (5.7 kDa), angiotensin II (1.1 kDa), 2-nitrobenzonitrile (2-NBN), and 2-nitrophloroglucinol (2-NPG) were purchased from Sigma Aldrich (St. Louis, MO). Pertussis toxin mutant, consisting of 6 subunits (one each of S1-S4, and two of S5; 26.2, 21.9, 21.9, 12.1, and $11.9 \mathrm{kDa}$, respectively) was purchased from List Biological Laboratories (Campbell, CA). Calmodulin was provided by the Proteomics Core Facility of WSU (Detroit, MI), using a protocol which involved extraction from E. coli, and stored in a $20 \mathrm{mM}$ Tris buffer at $\mathrm{pH} 7.5$ and $2.0 \mathrm{nM}$ ethylene glycol tetraacetic acid at $\mathrm{pH}$ 7.5. It was used without purification. LC-MS grade water, HPLC grade methanol (MeOH), HPLC grade acetonitrile (ACN), and glacial acetic acid were purchased from Fisher Scientific (Fairlawn, NJ). 3-Nitrobenzonitrile (3-NBN) was purchased from TCl America (Portland, OR). Myoglobin and cytochrome $\mathrm{C}$ were prepared by reconstituting the lyophilized powder in water 
then further diluted in $50 \%$ aqueous $\mathrm{MeOH}$ with $1 \%$ acetic acid to concentrations ranging from $50 \mathrm{nM}$ to $1 \mu \mathrm{M}$ and used without further purification. Lysozyme, ubiquitin, bovine insulin, and angiotensin II were prepared in $50 \%$ aqueous $\mathrm{ACN}$ with $1 \%$ acetic acid. Calmodulin extract was diluted in $50 \%$ aqueous $\mathrm{MeOH}$ with $1 \%$ acetic acid. 3-NBN and 2-NBN matrix solutions were both prepared to a concentration of $100 \mathrm{mg} / \mathrm{mL}$ in ACN. Prior to analysis, matrix and analyte solutions were mixed 1:1 (v:v) in a microcentrifuge tube and vortexed briefly (ca. 3 seconds). After mixing, 1 or $1.5 \mu \mathrm{L}$ of the matrix:analyte solution was drawn into the end of a disposable pipet tip, and allowed to crystallize for ca. 10 seconds on the end of the pipet tip upon which crystals were subsequently drawn into the aperture of the mass spectrometer by the pressure differential between atmosphere and the vacuum of the mass spectrometer.

A sample containing $40 \mu \mathrm{M}$ cholera toxin B-subunit in $50 \mathrm{mM}$ TRIS buffer ( $\mathrm{pH} 7.5$ ), 200 $\mathrm{mM} \mathrm{NaCl}, 1 \mathrm{mM}$ EDTA and $3 \mathrm{mM} \mathrm{NaN}_{3}$ was diluted 40 fold to a final protein concentration of 1 $\mu \mathrm{M}(1 \mathrm{pmol} / \mu \mathrm{L})$ and analyzed by MAI-MS. Pertussis toxin mutant was analyzed by MAI-MS from a sample containing $0.5 \mathrm{mg} / \mathrm{mL}$ of the protein in $10 \mathrm{mM}$ sodium phosphate buffer $(\mathrm{pH} 7.0)$ with $50 \mathrm{mM} \mathrm{NaCl}$. The final concentration of the protein after dilution was $1 \mu \mathrm{M}$. The mutations are present on the S1 subunit (R9K and E129A) to inactivate the enzymatic activity, making it safer to handle [21].

\section{Results and Discussion}

A direct comparison between MAI, without and with the use of a laser, is shown for lysozyme using different MAI matrices, previously described [4,22], and inlet tube temperatures

of $150{ }^{\circ} \mathrm{C}$ (Figure 1.A.) and $250{ }^{\circ} \mathrm{C}$ (Figure 1.B. and Figure S1). In MAI, the matrix:analyte crystals are physically introduced into the AP inlet without the use of a laser, whereas the use of a laser to ablate the sample into the inlet has been referred to as LSI and is shown in Scheme 1 
and Movie S1. A MAI matrix is crystallized together with the analyte on a glass plate, similar to MALDI sample preparation, and the glass plate is adhered to the atmospheric pressure inlet of an LTQ Velos mass spectrometer by the pressure differential so that the matrix:analyte crystals experience approximately the same sub-atmospheric pressure for MAI and LSI acquired using the same sample (Figure 1). At an inlet tube temperature of $150{ }^{\circ} \mathrm{C}$ using the 2-NBN matrix (Figure 1.A) the MAI total ion signal abundance is $1 / 3$ of the intensity of the LSI total ion signal abundance whereas at an inlet tube temperature of $250{ }^{\circ} \mathrm{C}$ the total ion signal abundance ratio of MAI and LSI is 8/1 (Figure 1.B) as shown in the total ion chronograms (TICs) (Figure 1.A.1 and 1.B.1). As visualized in the movie provided in the Supplementary file, MAI occurs when the glass plate is placed on the inlet tube connector (first signal in TIC) while the second signal in the TIC is with the laser. The relative ion intensities give an indication of the effectiveness of MAI without employing the laser, and especially with increasing the inlet tube temperature using the 2-NBN matrix. Performing the same experiment (Scheme 1), but with the matrix 2-NPG (a useful LSI matrix, even operating from high vacuum [22]), the sample sublimed rapidly without the use of a laser when exposed to vacuum condition at an inlet tube temperature of $150{ }^{\circ} \mathrm{C}$ (Figure S1); a laser was not needed at either 150 or $250{ }^{\circ} \mathrm{C}$. In all cases multiply charged ions are formed, keeping $\mathrm{m} / \mathrm{z}$ values well within the mass range of the mass spectrometer.

A direct comparison of MAI and LSI on the SYNAPT G2S shows elevated ion peak abundance using MAI (5.12E3) versus LSI (7.53E2) (Figure S2). We attribute this difference to a more efficient introduction of the matrix:analyte into the mass spectrometer inlet using MAI. In LSI, the laser beam can be focused to ablate only small areas providing high spatial resolution. The inclusion of the ion mobility spectrometry (IMS) notably increases the signal to noise ratio (Figure S2.I.B. and S2.II.B.).

Analyses from buffered protein solutions are applicable as is shown for positive mode measurements of calmodulin with charge states ranging from as high as +18 to as low as +5 , 
depending on instrumental conditions (Figure 2). It is also demonstrated that calmodulin is capable of being ionized in the negative ion mode by MAI, producing charge states between -18 and -8 (Figure S3). The added expense of lasers and safety requirements, as well as the higher background when using a laser, make MAI very attractive. For these reasons, the following studies were conducted in the Proteomics Core Facility at Wayne State University without the use of a laser.

The requirements for successful MAI are a proper matrix and temperature/pressure combination. Without instrumental modifications, the pressure of the experiment is usually fixed depending on whether the sample is introduced through the AP inlet or placed directly into vacuum. However, at least with the AP inlet, there is a range of temperatures that can be studied. An inlet temperature study on the Thermo Orbitrap Fusion shows the best ion abundance achieved for ubiquitin is at an inlet temperature of $100{ }^{\circ} \mathrm{C}$ (Figure S4). Deconvolution programs developed for ESI can be employed to convert multiply charged ions into the molecular weight (MW) of the analyte, as shown for calmodulin (Figure 2). Because of the ultra-high resolution of the Orbitrap Fusion used in this experiment (Figure 2C), the output of the protein mass measurement is with isotopic resolution when acquired with the resolution set to $\mathrm{m} / \Delta \mathrm{m} 450,000$ (50\% valley) at $\mathrm{m} / \mathrm{z} 400$. In addition, rapid acquisition of five proteins (ubiquitin, lysozyme, cytochrome c, calmodulin, and myoglobin) ranging in MW from 8.5 to 17 $\mathrm{kDa}$ is achieved within a minute (Figure $\mathbf{3}$ and Figure S5). The abundant highly charged protein ions are also readily accessible to be fragmented to allow sequence information to be obtained by using electron transfer dissociation (ETD), as is shown for ubiquitin charge state +12 obtained within $\sim 20$ seconds (Inset of Figure S6). Because of the ultra-high resolution, the charge states of ETD generated fragments of high-mass ions are deciphered by the program automatically. The high resolving power is also useful for direct analysis of small molecules from complex mixtures. MAI MS of the drug fexofenadine, in urine, and from blood wiped from skin 
surfaces is shown in Figure $\mathbf{S 7}$ and Figure 4, respectively. The background in the skin sample is likely from PEG used in body lotion. MAI produces few chemical background ions that are not sample related allowing the active ingredient of the drug to be readily detected.

An advantage of using MAI, especially relative to $\mathrm{ESI}$, is that high salt content is well tolerated. A buffered solution of the B-subunit of cholera toxin shows multiply protonated ions up to the charge state of +16 with essentially no chemical background on a SYNAPT G2 mass spectrometer (Figure 5). Even in the presence of high amounts of $\mathrm{NaCl}$ and phosphate buffer, the amount of salt adduction observed is minimal (Figure 6.A.). The IMS on this instrument allows the extraction of the mass spectra of the cholera toxin B-subunit at concentrations as low as $25 \mathrm{nM}$ (Figure S8) without the need for purification or concentration prior to analysis. A direct comparison of the buffered solution of B-subunit of cholera toxin using different ionization methods show ion abundances readings (Figure 6) for the most abundant charge state: $\left([\mathrm{M}+9 \mathrm{H}]^{9+}\right)$ of $1.47 \mathrm{e} 5$ for $\mathrm{MAI},\left([\mathrm{M}+8 \mathrm{H}+\mathrm{Na}]^{9+}\right)$ of $4.57 \mathrm{e} 3$ for $\mathrm{ESI}$, and $\left([\mathrm{M}+\mathrm{H}]^{+}\right)$of $7.20 \mathrm{e} 4$ for MALDI (please note that this data was acquired on two different mass spectrometers). While both MAI (Figure 6A) and ESI (Figure 6B) provide predominantly the protonated multiply charged ions, ESI has undesired interference of sodium adduction. The MALDI-TOF MS results (Figure 6C) show the typical broad protein signals and an ion associated with matrix adduction. These results indicate that MAI MS is sensitive and robust towards salt and sample additives such as EDTA and sodium azide $\left(\mathrm{NaN}_{3}\right)$.

Another example of MAI for a natural protein is shown for the pertussis toxin, a bacterial toxin that exists as a $117 \mathrm{kDa}$ hexameric protein complex made up of 6 subunits, denoted as S1 - S5 with the S5 subunit present in two copies (Figure 7). Analyte solvent conditions influence the analysis using the same matrix, 3-NBN, and the same instrument acquisition conditions (Figure 7). From aqueous solution conditions one detects preferentially the S4 subunits of bacterial toxin with charge states of +12 to +6 (Figure 7.A.). The addition of methanol and 
acetic acid to the solvent showed a decrease in the ratio of protonated:sodium adducted signals compared to the preparation in water. Using the acidified methanol-water solvent conditions, the MAI mass spectrum shows all of the subunits present (Figure 7.B.), with the $\$ 4$ subunit being the most abundant ion. The observed $\mathrm{m} / \mathrm{z}$ values for each subunit present in the mass spectrum are tabulated in Table S1. All of the species exist predominantly as multiply protonated ions, although some additional lower abundant peaks are observed due to the presence of sodiumadducted ions as well. Analysis of the B-subunit of pertussis toxin acquired using a MALDI-TOF mass spectrometer [23] resulted in singly charged ions of the S2 and S3 subunits (MW ca. 21.9 $\mathrm{kDa}$ ), differing in mass by ca. $100 \mathrm{Da}$, which could not be resolved at such a high $\mathrm{m} / \mathrm{z}$ because of the width of the protein signals associated with typical MALDI-TOF MS analysis [24,25]. With MAI however, multiple charging shifts the observed $\mathrm{m} / \mathrm{z}$ towards the lower mass range for higher mass resolution measurements [4], allowing both the S2 and S3 subunits to be resolved by $m / z$ using high resolution measurements (Figure S7.B.).

\section{Conclusion}

The results presented here show the potential of a new ionization method for analysis of nonvolatile biological compounds ionized directly from surfaces with (LSI) and without (MAI) the use of a laser. The new ionization methods lead to highly efficient ionization even for nonvolatile samples from 'dirty' conditions and, when performed on high-performance mass spectrometers, allow rapid deciphering of complex samples. Analyte solution conditions factor into the ion abundance of buffered and salty samples such as those of the membrane-associated bacterial toxins. The results presented here show that MAI and LSI have advantages relative to ESI and MALDI even though the experiments are carried out on instruments designed for these latter techniques. The success of LSI and MAI is related to the search of matrices with increasing volatility under vacuum conditions $[5,26]$. 


\section{Acknowledgement}

This work was made possible by the generous support from NSF CAREER 0955975 , NSF CHE-1411376, DuPont Young Professor Award, Eli Lilly Young Investigator Award in Analytical Chemistry, Waters Center of Innovation Program, Thermo Scientific, and WSU Schaap Faculty Scholar (to ST) as well as grants to support the Core (and to PMS) P30 ES020957, P30 CA022453 and S10 OD010700. We would like to specifically thank Drs. Alexander Makarov and Jean-Jacques Dunyach for their input with the Orbitrap Fusion (Thermo). 


\section{References}

[1] A.J. Link, J. Eng, D.M. Schieltz, E. Carmack, G.J. Mize, D.R. Morris, B.M. Garvik, J.R. Yates, Direct analysis of protein complexes using mass spectrometry, Nat. Biotech 17 (1999) 676-682. [2] J.P. Overington, B. Al-Lazikani, A.L. Hopkins, How many drug targets are there?, Nat. Rev. Drug Discov. 5 (2006) 993-996.

[3] E.D. Inutan, S. Trimpin, Matrix assisted ionization vacuum (MAIV), a new ionization method for biological materials analysis using mass spectrometry, Mol. Cell. Proteomics 12 (2013) 792796.

[4] S. Trimpin, E.D. Inutan, Matrix assisted ionization in vacuum, a sensitive and widely applicable ionization method for mass spectrometry, J. Am. Soc. Mass Spectrom. 24 (2013) 722-732.

[5] S. Trimpin, C.A. Lutomski, T.J. El-Baba, D.W. Woodall, C.D. Foley, C.D. Manly, B. Wang, C.W. Liu, B.M. Harless, R. Kumar, L.F. Imperial, E.D. Inutan, Magic matrices for ionization in mass spectrometry, Int. J. Mass Spectrom. 377 (2015) 532-545.

[6] R.D. Smith, J.A. Loo, C.G. Edmonds, C.J. Barinaga, H.R. Udseth, New developments in biochemical mass spectrometry: electrospray ionization, Anal. Chem. 62 (1990) 882-899. [7] M. Sarbu, F. Zhu, J. Peter-Katalinic, D.E. Clemmer, A.D. Zamfir, Application of ion mobility tandem mass spectrometry to compositional and structural analysis of glycopeptides extracted from the urine of a patient diagnosed with Schindler disease, Rapid Commun. Mass Spectrom. 29 (2015) 1929-1937.

[8] C.J. Tu, Q.H. Sheng, J. Li, D.J. Ma, X.M. Shen, X. Wang, Y. Shyr, Z.P. Yi, J. Qu, Optimization of search engines and postprocessing approaches to maximize peptide and protein identification for high-resolution mass data, J. Proteome Res. 14 (2015) 4662-4673. 
[9] M.J. Sweredoski, A. Moradian, M. Raedle, C. Franco, S. Hess, High Resolution Parallel Reaction Monitoring with Electron Transfer Dissociation for Middle-Down Proteomics Anal. Chem. 87 (2015) 8360-8366.

[10] S. Trimpin, E.D. Inutan, New ionization method for analysis on atmospheric pressure ionization mass spectrometers requiring only vacuum and matrix assistance, Anal. Chem. 85 (2013) 2005-2009.

[11] S. Trimpin, E.D. Inutan, T.N. Herath, C.N. McEwen, Laserspray lonization - A new atmospheric pressure MALDI method for producing highly charged gas-phase ions of peptides and proteins directly from solid solutions, Mol. Cell. Proteomics 9 (2010) 362-367.

[12] S. Trimpin, E.D. Inutan, T.N. Herath, C.N. McEwen, Matrix-assisted laser desorption/ionization mass spectrometry method for selectively producing either singly or multiply charged molecular ions, Anal. Chem. 82 (2010) 11-15.

[13] S. Trimpin, "Magic" Ionization Mass Spectrometry. J. Am. Soc. Mass Spectrom., 2015, DOI: 10.1007/s13361-015-1253-4. (published on-line ahead of print)

[14] S. Trimpin, B. Wang, E.D. Inutan, J. Li, C.B. Lietz, V.S. Pagnotti, A.F. Harron, D. Sardelis, C.N. McEwen, A mechanism for ionization of nonvolatile compounds in mass spectrometry: considerations from MALDI and inlet ionization, J. Am. Soc. Mass Spectrom. 23 (2012) 16441660.

[15] T.L. Constantopoulos, G.S. Jackson, C.G. Enke, Effects of salt concentration on analyte response using electrospray ionization mass spectrometry, J. Am. Soc. Mass Spectrom. 10 (1999) 625-634.

[16] D.-C. Moon, J.A. Kelley, A simple desalting procedure for fast atom bombardment mass spectrometry, Biomed. Environ. Mass 17 (1988) 229-237.

[17] M.G. Pluskal, Microscale sample preparation, Nat. Biotech. 18 (2000) 104-105. 
[18] S. Koster, E. Verpoorte, A decade of microfluidic analysis coupled with electrospray mass spectrometry: An overview, Lab on a Chip 7 (2007) 1394-1412.

[19] W.E. Haskins, Z. Wang, C.J. Watson, R.R. Rostand, S.R. Witowski, D.H. Powell, R.T. Kennedy, Capillary LC-MS2 at the attomole level for monitoring and discovering endogenous peptides in microdialysis samples collected in vivo, Anal. Chem. 73 (2001) 5005-5014.

[20] D.W. Woodall, B. Wang, E.D. Inutan, S.B. Narayan, S Trimpin, High-throughput characterization of small and large molecules using only a matrix and the vacuum of a mass spectrometer. Anal. Chem. 87 (2015) 4667-4674.

[21] M. Pizza, A. Covacci, A. Bartoloni, M. Perugini, L. Nencioni, M.T. De Magistris, L. Villa, D. Nucci, R. Manetti, M. Bugnoli, F. Giovanoni, R. Olivieri, J.T. Barbieri, H. Sato, R. Rappuoli, Mutants of Pertussis Toxin Suitable for Vaccine Development. Science 246 (1989) 497-500. [22] S. Trimpin, Y. Ren, B. Wang, C.B. Lietz, A.L. Richards, D.D. Marshall, E.D. Inutan, Extending the laserspray ionization concept to produce highly charged ions at high vacuum on a time-of-flight mass analyzer, Anal. Chem. 83 (2011) 5469-5475.

[23] B. Liang, Y. Ju, J.R. Joubert, E.J. Kaleta, R. Lopez, I.W. Jones, H.K. Hall Jr, S.N. Ratnayaka, V.H. Wysocki, S.S. Saavedra, Label-free detection and identification of protein ligands captured by receptors in a polymerized planar lipid bilayer using MALDI-TOF MS, Anal. Bioanal. Chem. 407 (2015) 2777-2789.

[24] S. Niu, W. Zhang, B.T Chait, Direct Comparison of Infrared and Ultraviolet Wavelength Matrix-assisted laser Desorption/lonization Mass Spectrometry of Proteins, J. Am. Soc. Mass Spectrom. 9 (1998) 1-7.

[25] S. Trimpin, M.L. Deinzer, Solvent-free MALDI-MS for the Analysis of a Membrane Protein via the Mini Ball Mill Approach: Case Study of Bacteriorhodopsin, Anal. Chem. 79 (2007) 71-78. 
[26] C.N. McEwen, B.S. Larsen, S. Trimpin, S., Laserspray ionization on a commercial atmospheric pressure-MALDI mass spectrometer ion source: selecting singly or multiply charged ions, Anal. Chem. 82 (2010) 4998-5001. 


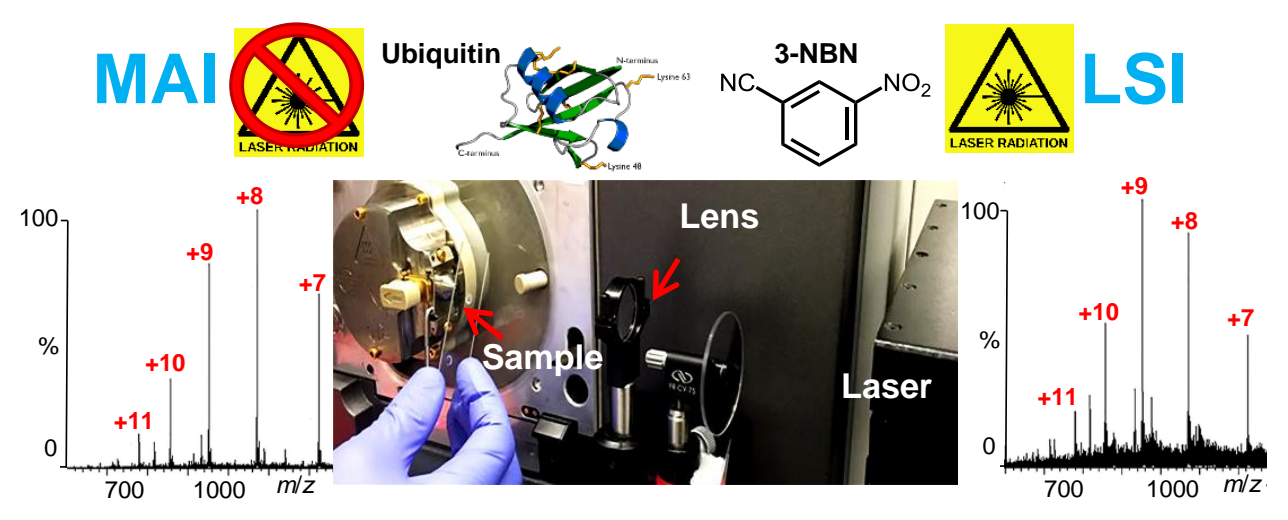

Graphical Abstract

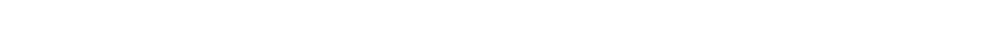


Figure 1

Figure 1
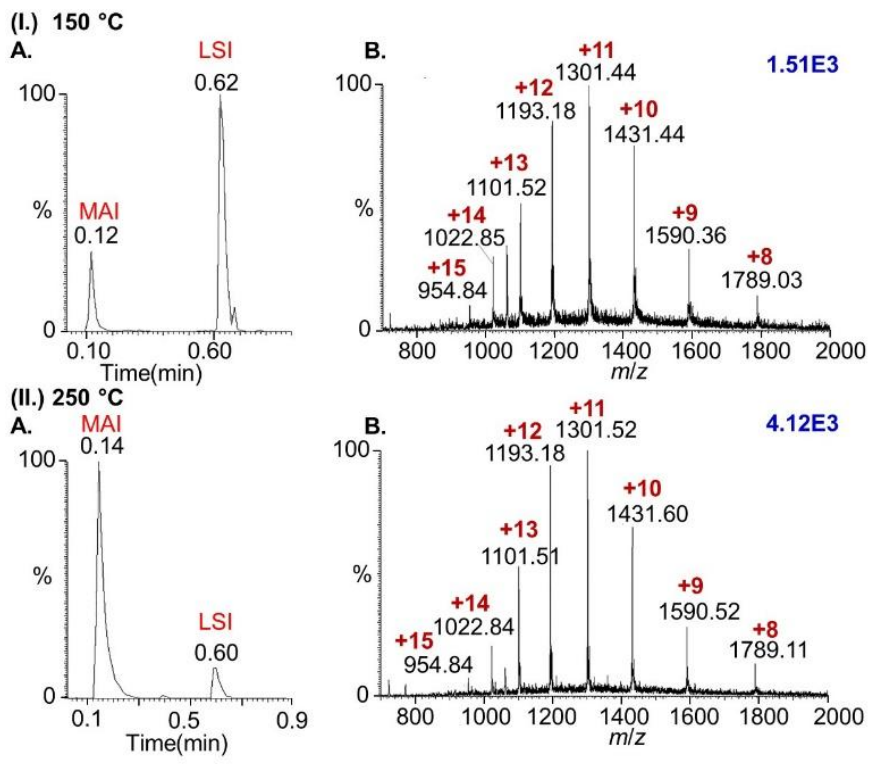


\section{Figure 2}
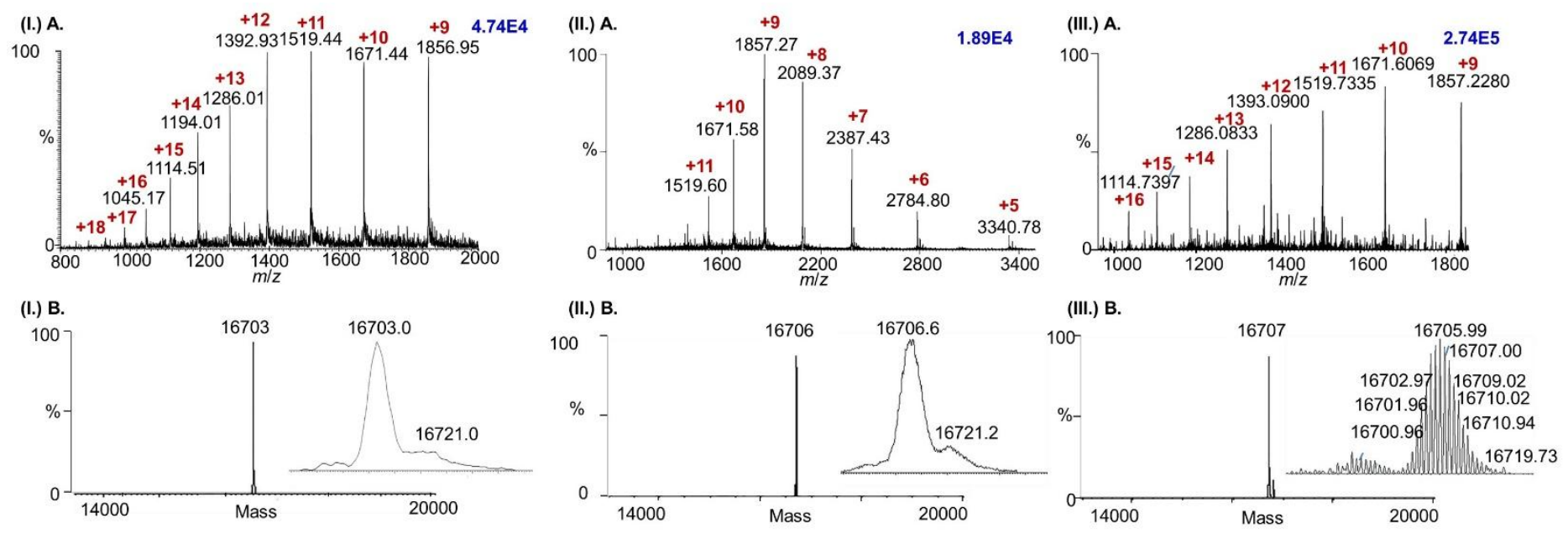

\section{Figure 2}


Figure 3
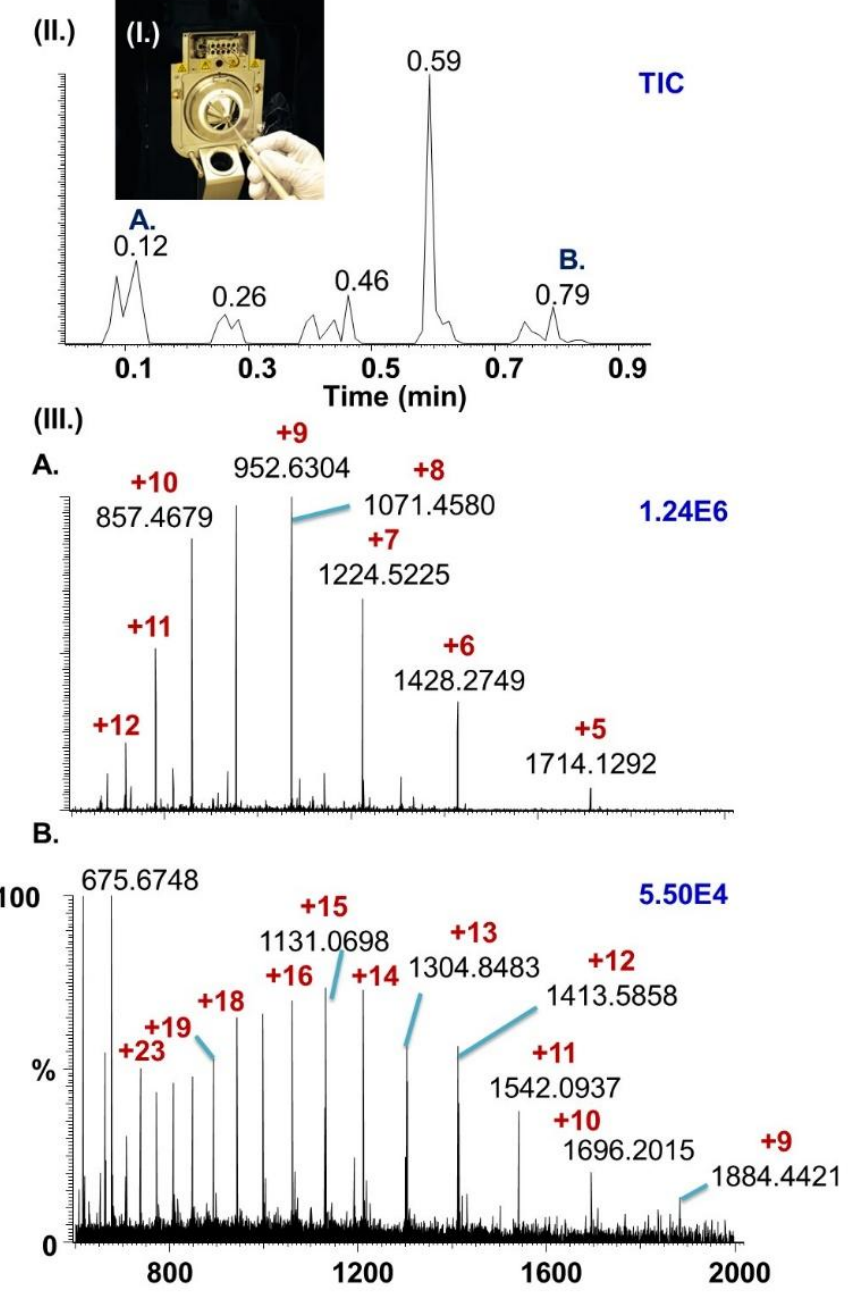


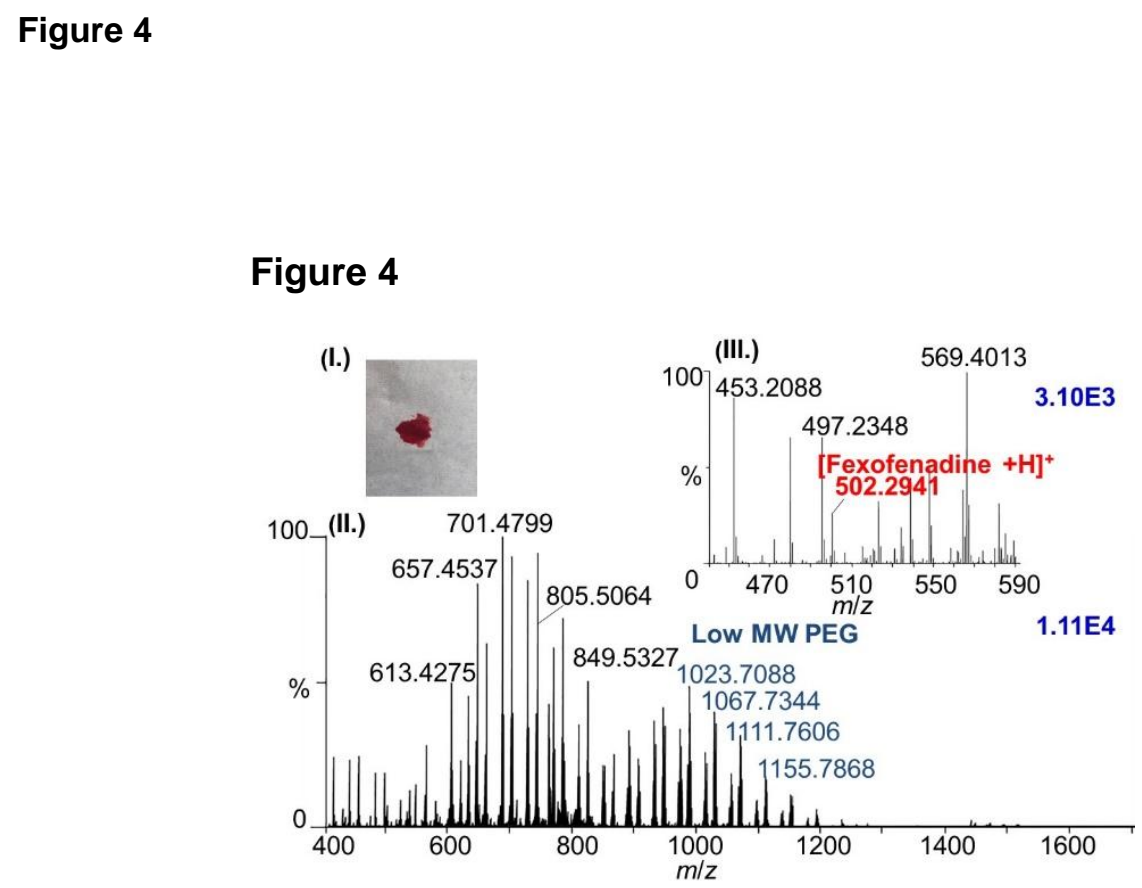

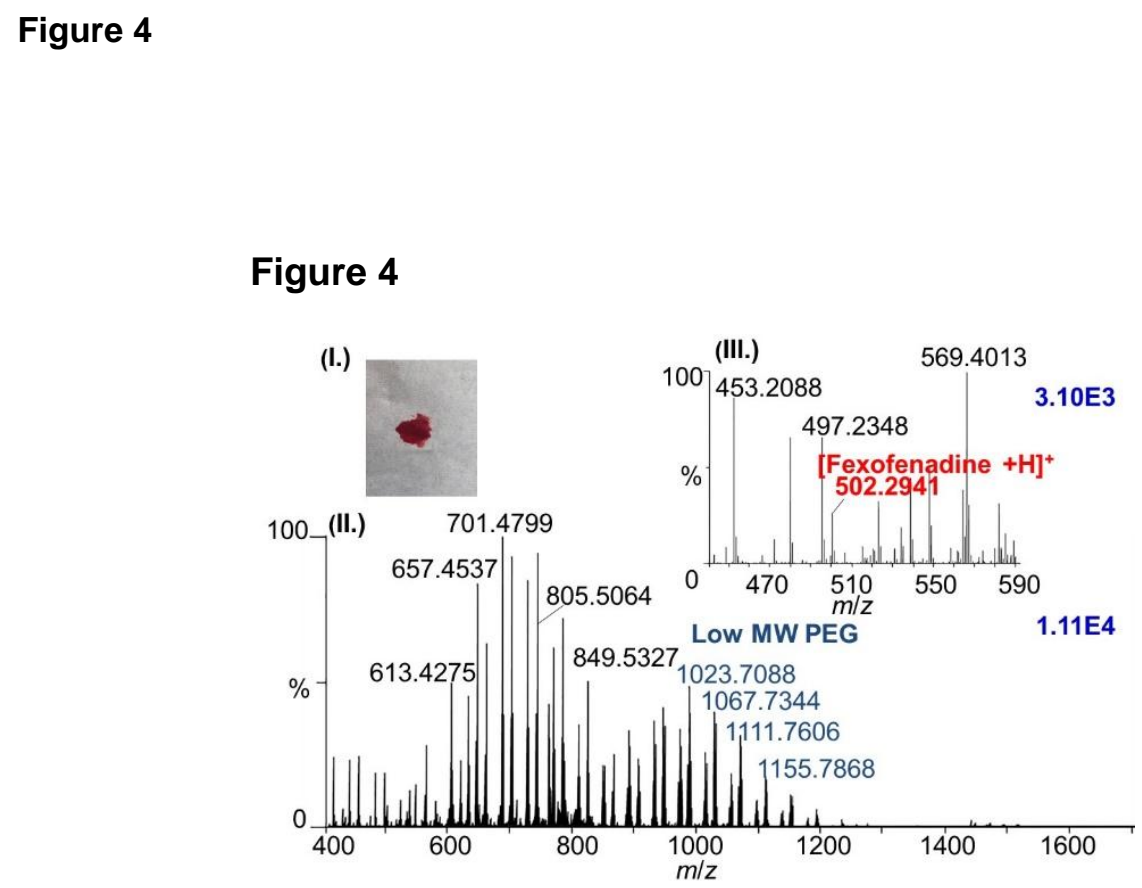
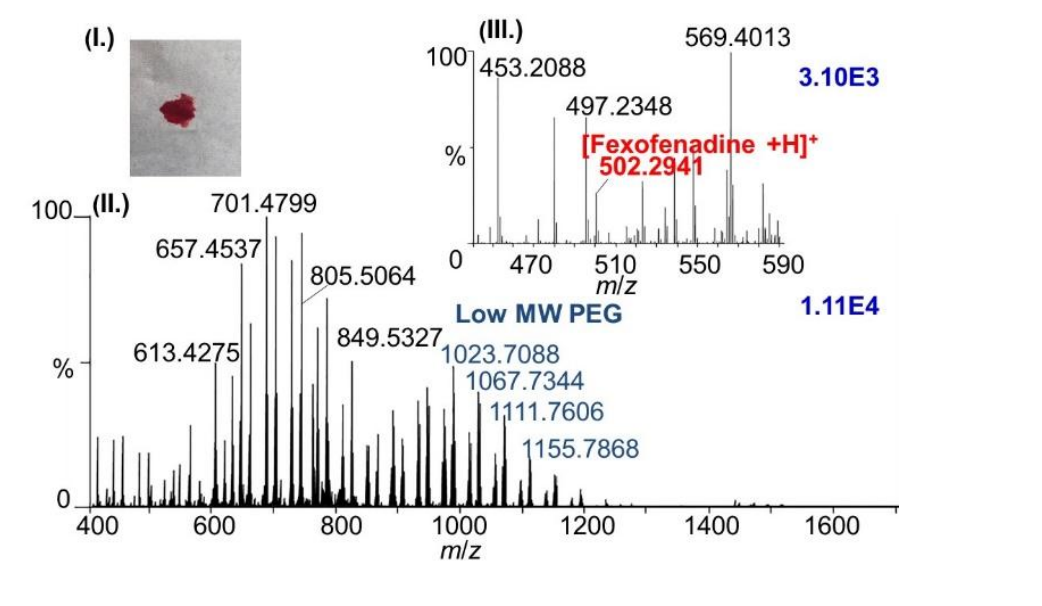

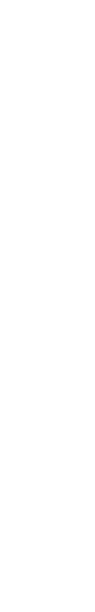

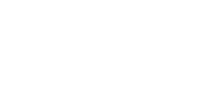
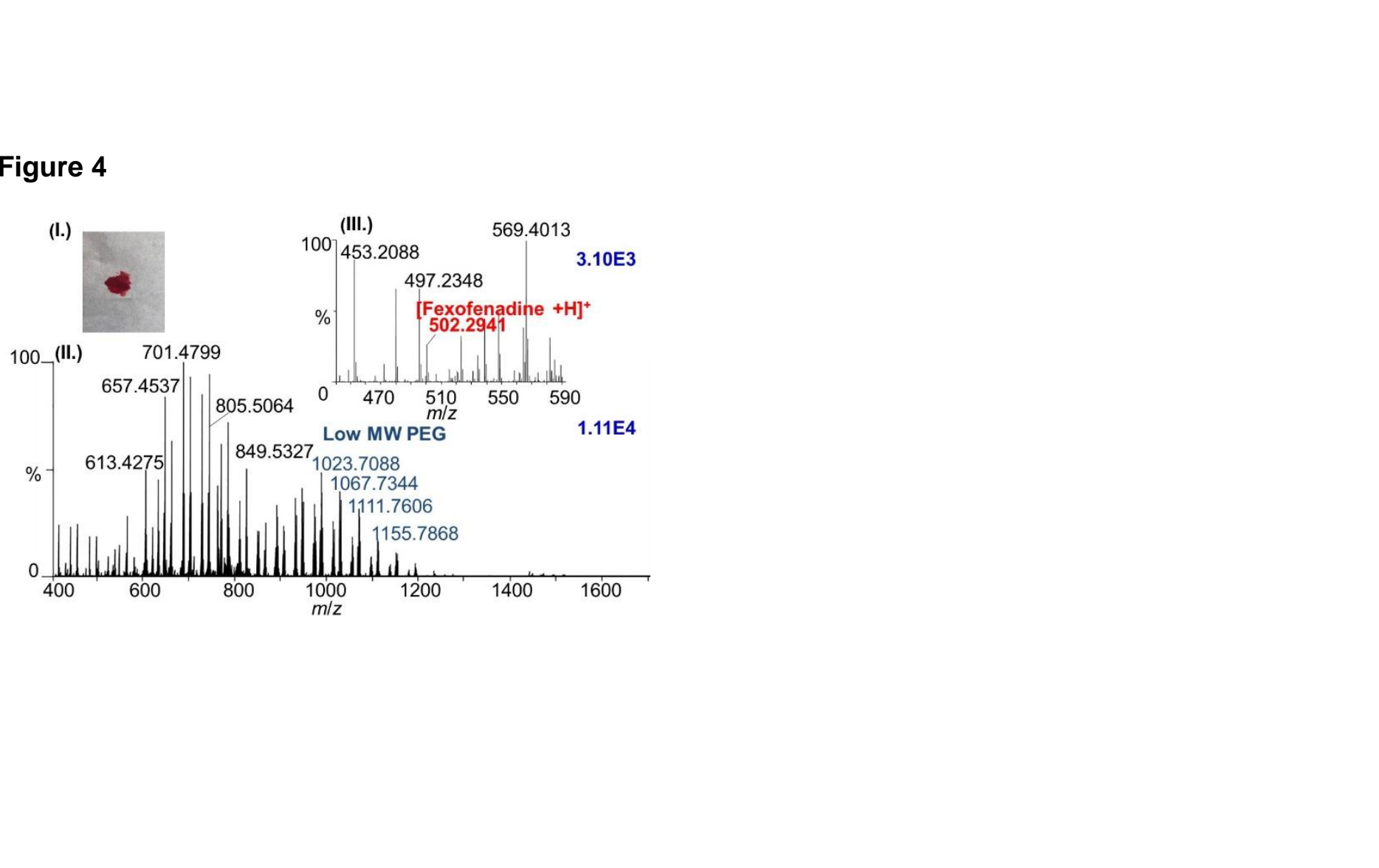
Figure 6

\section{Figure 6}

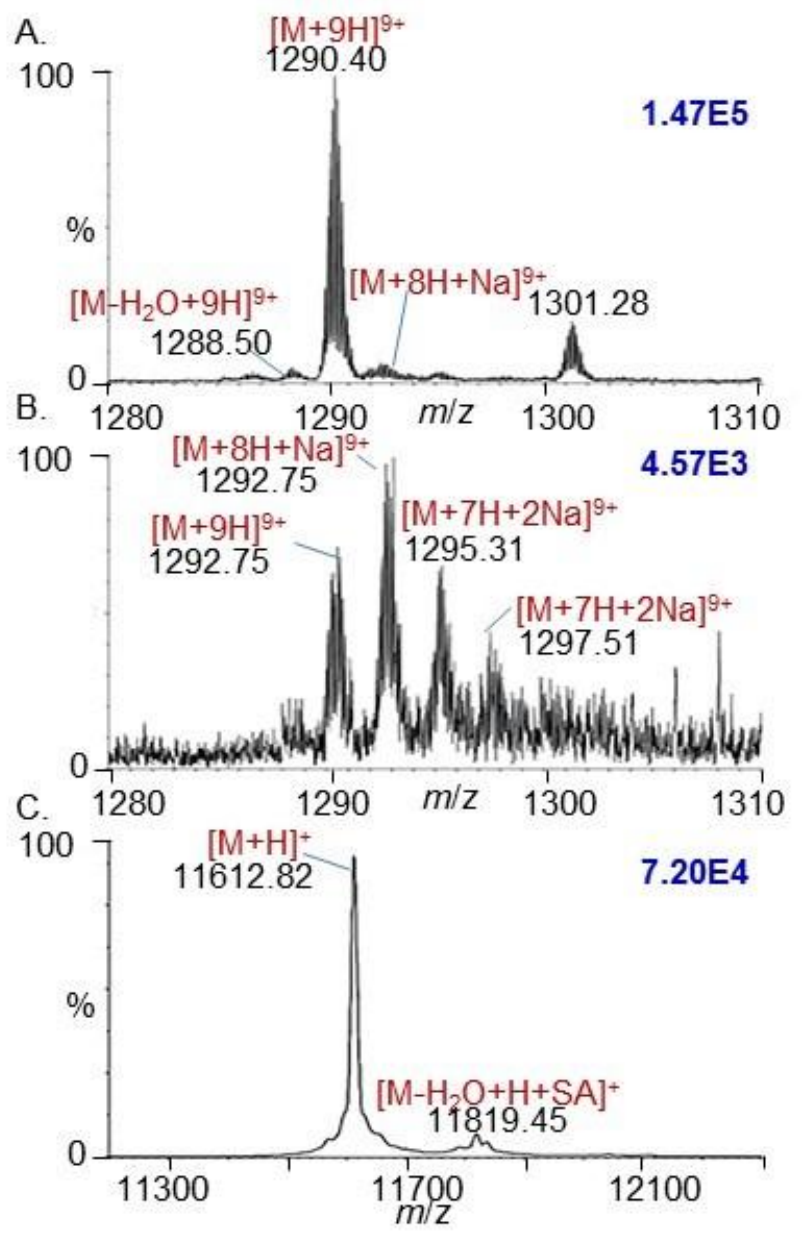


Figure 7

Figure 7

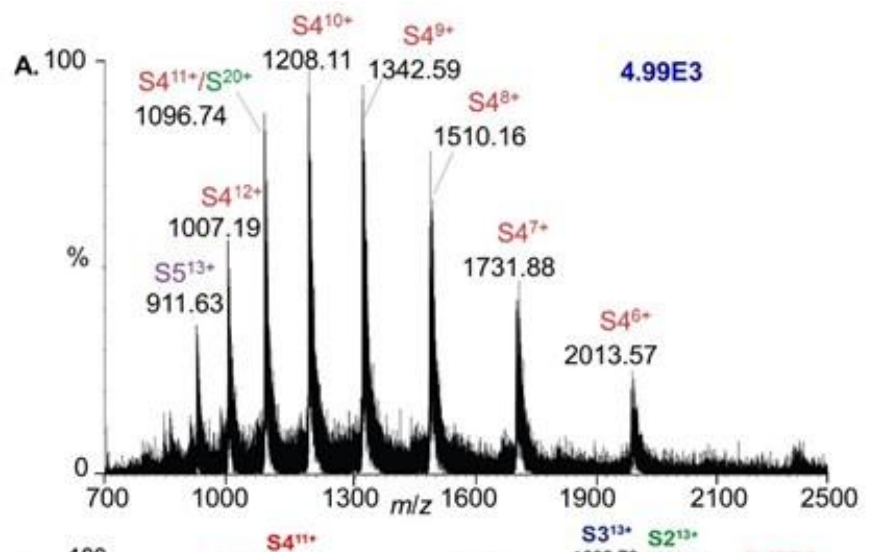

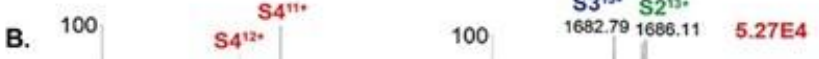

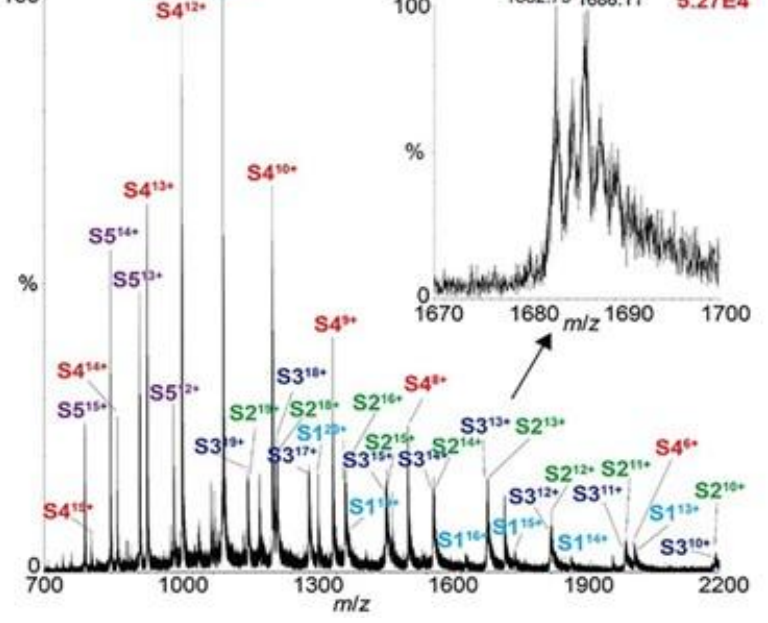

